\title{
Ion-Current Downstream of a Carbon Dioxide Helicon Double Layer
}

\author{
Christine Charles, Rhys Hawkins, and Rod Boswell
}

\begin{abstract}
Energy-resolved ion measurements in a carbon dioxide helicon radio-frequency plasma containing an oblique current-free double layer are obtained using a retarding field energy analyzer for standard operating conditions (i.e., 0.4 mTorr, $250 \mathrm{~W}$, and $140 \mathrm{G}$ ). Two ion populations corresponding to thermal ions and an energetic ion beam, respectively, are observed and spatially mapped downstream of the double layer, which is in the region of diverging field lines. The measurements are mirrored about the main reactor axis using cylindrical geometry, and a 3-D image is generated.
\end{abstract}

Index Terms-Ion beams, plasma sources.

$\mathbf{L}$ OW-PRESSURE magnetically expanding radiofrequency (RF) plasmas can contain electric double layers, which lead to particle acceleration [1], [2]. These have been studied experimentally, analytically, and by computer simulation in the context of electric-propulsion applications and astrophysical plasmas [3], [4]. Here, a current-free double layer is generated in a helicon source operating with a carbon dioxide gas [5]. For this gas, the double layer protudes out of the plasma source tube, allowing for a detailed spatial mapping of the ion current using an energy analyzer. Here, we present an image obtained from the analysis of ion-current measurement downstream of the U-shaped double layer.

The helicon double-layer source is mounted on the Chi Kung chamber (see Fig. 1), and the system has been previously described [4]-[6]. The plasma source consists of a pyrex source tube surrounded by an RF antenna and two axial solenoids. The $\mathrm{z}$-axis is the main axis, the source/chamber interface is located at $z=30 \mathrm{~cm}$, and the low-potential edge (passing by $(x, z)=(0,34) \mathrm{cm}$ where the field is about $39 \mathrm{G})$ of the current-free double layer is shown by the parabolic solid line in Fig. 1 [5]. The high-potential side is to the left of the parabola, and ions accelerated in the double-layer potential drop $(10 \mathrm{~V}$ at $250 \mathrm{~W}$ and $0.4 \mathrm{mTorr}$ ) form a beam, which propagates in the diverging magnetic field from left to right. The total ion current $I_{\text {total }}$ and the ion beam current $I_{\text {beam }}$ have been measured experimentally in the region defined between $z=30-45 \mathrm{~cm}$ and $x=0--15 \mathrm{~cm}$ using the dogleg retarding field energy analyzer previously described [7], [8] (see Fig. 1).

Manuscript received October 16, 2010; revised April 6, 2011; accepted May 8, 2011. Date of publication June 23, 2011; date of current version November 9, 2011.

C. Charles and R. Boswell are with the Space Plasma, Power and Propulsion Laboratory, Research School of Physics and Engineering, Australian National University, Canberra, ACT 0200, Australia.

R. Hawkins is with the Supercomputer Facility, Australian National University, Canberra, ACT 0200, Australia.

Digital Object Identifier 10.1109/TPS.2011.2155087

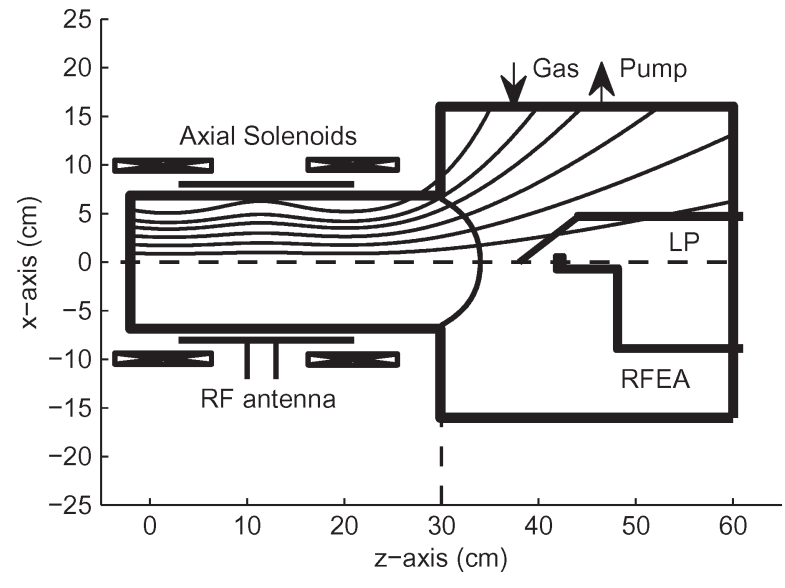

Fig. 1. Schematic of the Chi Kung experimental setup.

The mapping of the total current $I_{\text {total }}$ has already shown the presence of high-density ion conics correlated with the first "open" field line originating from the helicon source and with an equipotential contour giving a connection path from the source to the grounded chamber [9]. The mapping of $I_{\text {beam }}$ has also been performed previously by using a two-Gaussian deconvolution method [7], [8] where the low-energy Gaussian corresponds to the population of thermal ions and the highenergy Gaussian corresponds to the ion-beam population. Ions contributing to the beam component have energy values in the region of $10 \mathrm{eV}$, whereas ions in the thermal group have energy values of less than $1 \mathrm{eV}$ (i.e., Gaussian broadening by a few volts due to RF sheath and collisional effects are present). On the first approximation, the population of thermal ions can be estimated by calculating the difference between the total and ion-beam currents $I_{\text {thermal }} \sim\left(I_{\text {total }}-I_{\text {beam }}\right)$.

Fig. 2 is a 3-D image of the low-potential edge of the double layer (i.e., the light-blue dome), the ion-beam current (i.e., the yellow-to-red flame of decreasing intensity due to ion-neutral collisions along the expansion) and the thermal ion current (i.e., black and dark-blue "conics" with a pink/purple central region of maximum current).

Fig. 2 suggests that the divergence of the ion beam stays reasonably low (about $13^{\circ}$ for $80 \%$ of the beam shown by a solid black line).

It should be noted that the maximum magnetic field line divergence is also the region of minimum accuracy. In this region, the energy analyzer, which has an acceptance angle of a few tens of degrees [8], is always directed along a horizontal axis parallel to the z-axis, i.e., not aligned with the electric field of the double layer. 


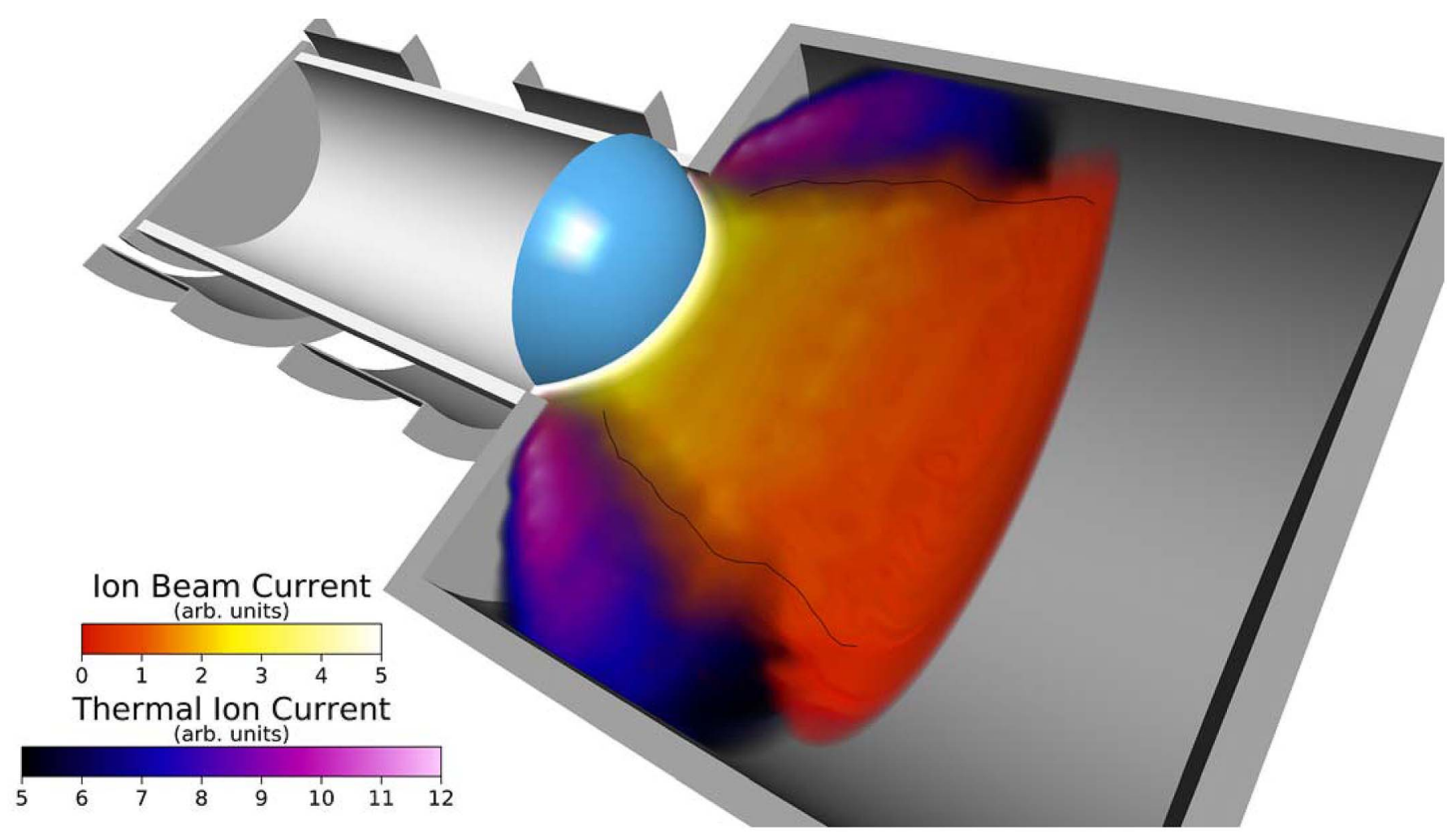

Fig. 2. Low-potential edge of the carbon dioxide double layer (light blue dome), ion beam current $I_{\text {beam }}$ (white to yellow to red flame), and thermal ion current $I_{\text {thermal }}$ (purple to blue to black "conics") measured in the Chi Kung plasma reactor using the energy analyzer.

In conclusion, an image of ion populations downstream of an oblique current-free double layer is computed from an experimental mapping. This paper has shown the importance of the diverging-magnetic-field configuration on the ion populations, with a maximum effect on the thermal ions and a minimum effect on the ion beam and its detachment from the magnetic field.

\section{REFERENCES}

[1] M. A. Raadu, "The physics of double layers and their role in astrophysics," Phys. Rep., vol. 178, no. 2, pp. 25-97, Apr. 1989.

[2] C. Charles, "A review of recent laboratory double layer experiments," Plasma Sources Sci. Technol., vol. 16, no. 4, p. R1-25, Nov. 2007.

[3] M. D. West, C. Charles, and R. W. Boswell, "Testing a helicon double layer thruster immersed in a space-simulation chamber," J. Propulsion Power, vol. 24, no. 1, pp. 134-141, Jan./Feb. 2008.
[4] R. W. Boswell, E. Marsch, and C. Charles, "The current-free electric double layer in a coronal magnetic funnel," Astrophys. J., vol. 640, no. 2, pp. L199-L202, Apr. 2006.

[5] C. Charles, R. W. Boswell, and R. Hawkins, "Oblique double layers: A comparison between Terrestrial and Auroral measurements," Phys. Rev. Lett., vol. 103, no. 9, p. 095001, Aug. 2009.

[6] C. Charles and R. W. Boswell, "The magnetic-field-induced transition from an expanding plasma to a double layer containing expanding plasma," Appl. Phys. Lett., vol. 91, no. 20, pp. 201505-1-201505-3, Nov. 2007.

[7] C. Charles, R. W. Boswell, and M. A. Lieberman, "Xenon ion beam characterization in a helicon double layer thruster," Appl. Phys. Lett., vol. 89, no. 26, pp. 261503-1-261503-3, Dec. 2006.

[8] C. Charles and R. W. Boswell, "Laboratory evidence of a supersonic ion beam generated by a current-free "helicon" double-layer," Phys. Plasmas, vol. 11, no. 4, pp. 1706-1714, Apr. 2004.

[9] C. Charles, "High density conics in a magnetically expanding helicon plasma," Appl. Phys. Lett., vol. 96, no. 5, pp. 051502-1-051502-3, Feb. 2010. 